\title{
Editorials
}

\section{Mental illness and resilience among sexual and gender minority refugees and asylum seekers}

It is well documented that refugees and asylum seekers (RAS) experience high levels of mental illness. This is also true of sexual and/or gender minority (SGM) individuals. Based on a literature summary, we present the current research on mental illness in individuals who experience the 'multiple minority stress' of being both SGM individuals and RAS.

In high-income countries worldwide, hardening attitudes towards RAS are being spurred on by rising rates of international migration combined with policies such as those of the 'hostile environment' and 'zero tolerance'. As individuals at the intersection of multiple minority identities, SGM RAS arriving in countries such as the UK not only have to navigate this complex sociopolitical and cultural context, but also have to navigate specific oppressions, such as reports of frequent rejection of asylum claims made in the UK on the basis of persecution for sexual orientation or gender identity. It is in this context that we present this research, aiming to draw attention to the existence of the SGM RAS population, their particular needs and vulnerabilities, and to highlight factors making them susceptible and resilient to mental illness.

In this report, the term ' $S G M$ ' is chosen over 'LGBTQIA', as 'lesbian', 'gay', 'bisexual', 'transgender', 'queer', 'intersex' and 'asexual' are terms originating in the Western world which may not be used or identified with by SGM individuals from other cultures.

\section{MENTAL ILLNESS IN SEXUAL AND/OR GENDER MINORITY REFUGEES AND ASYLUM SEEKERS}

To date, six studies have been published on mental illness in SGM RAS. ${ }^{1-6}$ All six were based in North America and were published in 2016-2017. No research focused on SGM RAS in refugee camps or detention centres. The research focused on cisgender male and female sexual minorities la cisgender person being a person whose gender identity corresponds with the gender they were assigned at birth), with little being focused on transgender women, and none on transgender men, other gender minorities or intersex people.

Common mental health problems reported in these studies included depression, anxiety, post-traumatic stress

having multiple minority identities has a cumulative impact on the prevalence of mental illness in this population.

disorder (PTSD), suicidality, and alcohol and substance misuse issues. The prevalence of depression in SGM RAS ranged from $76.0-93.0 \%, 3,6$ and of anxiety ranged from 28.0-60.0\%.3.6 Particularly striking was the prevalence of PTSD, reportedly as high as $70.0-100.0 \%, 3,6$ and current or past suicidality, as high as $56.0-72.1 \%$. $^{3,4}$ Prevalence rates of alcohol and substance misuse issues ranged from 2.0-36.0\%. Compared to non-SGM RAS or nonRAS SGM populations, ${ }^{7.8}$ these rates are considerably higher, suggesting that having multiple minority identities has a cumulative impact on the prevalence of mental illness in this population.

A key risk factor in the development of mental illness in SGM RAS is their experience of discrimination and/or violence in their sending countries, from community members and the state. $2,3,6,9$ Furthermore, while moving to a country perceived as more tolerant to SGM identities may be an important 'pull factor' for some SGM RAS, sexual and particularly gender minority people are still at risk of experiencing discrimination and violence post-migration. ${ }^{3,10}$ This includes structural violence perpetrated by government immigration determination processes in receiving countries, during which SGM RAS often have restricted access to health, social care, and employment rights, and also have to navigate the ever-present possibility of rejection and return to the countries they have fled. ${ }^{10}$ SGM RAS may additionally be required to 'prove' their SGM identity in order to successfully claim asylum on this basis. This not only ignores cultural differences in SGM identity formation and expression, but also forces these individuals to repeatedly 'come out' to border officials, lawyers, doctors, and social workers, which may remind them of past traumas and risk re-exposing them to homophobia and transphobia in their receiving country. ${ }^{11}$ Furthermore, SGM individuals from countries where SGM populations are victimised or even criminalised may be used to hiding their true sexuality or gender identity and therefore may struggle to 'evidence' them, having perhaps never had a partner of their chosen gender or publicly belonged to an SGM organisation or community.

It is important not to allow the discussion of mental illness in SGM RAS to be dominated by a negative narrative only. SGM RAS have also been shown to be enormously resilient, with key resilience factors including staying hopeful and positive, accessing social support, making use of available services, giving back to the community, and 'doing what it takes'? Access to community and legal services is particularly crucial in terms of accessing healthcare, food, financial assistance, housing, and information on the refugee determination process. ${ }^{2}$ Spiritual upkeep is helpful to some SGM RAS, though religious institutions can also be perpetrators of pre- and post-migration homophobic and transphobic violence. 2,9 SGM RAS-specific support groups may also improve the mental health of this population. ${ }^{5}$

\section{THE ROLE OF GENERAL PRACTICE}

Access to primary healthcare can help SGM RAS who experience or are at risk of mental illness to be supported and 


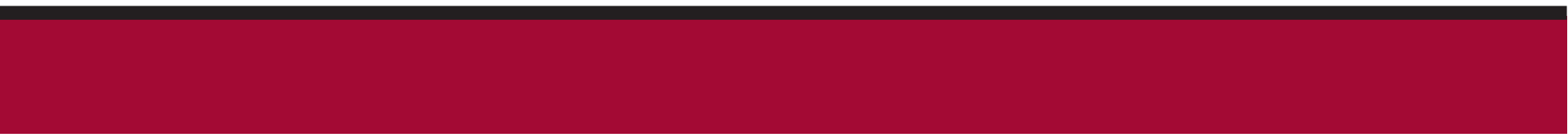

\section{"... as many as half of the RAS population in the UK never try to access a GP.'}

managed in the community and promote their resilience to further mental health problems. However, as many as half of the RAS population in the UK never try to access a GP, due to concerns around the cost of care, administrative barriers related to not having identification or proof of address, or fear of being arrested and deported. ${ }^{12}$ GP practices can improve their accessibility by clearly advertising information, in a variety of languages, on how they operate, registration requirements, and what services are free to access. Useful resources for practices can be accessed through the humanitarian organisation, Doctors of the World, who provide information and guidance on how practices can become 'safe surgeries' for excluded migrant patients.

Healthcare professionals are also well placed to advocate for better care, for example, encouraging their professional bodies and social networks to support and uphold the rights of all international migrants to have their primary care needs met in an inclusive and affordable healthcare system. One example of this would be through challenging recent government pressures on GP practices and emergency departments to impose identification checks on migrant patients before allowing them access to care. One example of campaigns of this nature is the Docs Not Cops campaign (http://www. docsnotcops.co.uk/), which uses slogans such as: 'We treat patients not passports - I am a doctor, not a border guard'.

SGM individuals may be unwilling to disclose their SGM identity to their GP due to fear of discrimination or receiving poorer quality care. ${ }^{13}$ To encourage SGM RAS to disclose their sexual orientation and/or gender identity, GP practices can ensure that they have signs informing patients that they will be treated with respect regardless of SGM identity, provide opportunities to disclose SGM identity on registration forms, and use non-heteronormative language in consultations such as asking patients about their 'partners' as opposed to their 'boyfriend/girlfriend' or 'husband/wife'.

SGM RAS are also less likely to seek support from their diaspora community due to fear of repeated victimisation. ${ }^{10}$ When GP practices are referring SGM RAS on to specialist support services, it is important to consider that these individuals may not feel safe in SGM- or migrant-specific services, unless said services are openly inclusive to those with multiple minority identities.

\section{CONCLUSION}

SGM individuals and RAS face ongoing stigma, discrimination and violence. A limited body of relatively recent research on the needs of SGM RAS underlines 'multiple minority stress' as key in contributing to the high rates of depression, anxiety, suicidality, PTSD and alcohol and substance misuse issues in this population. GP practices can play an important role in facilitating this population's access to primary care and appropriate mental health services. Although the SGM RAS population in the UK is hidden and requires outreach and trust, improving their access to such services has been shown to be key in strengthening their resilience to mental illness.

At a time of exhausting GP workloads and the 'hostile environment', the needs of patients with multiple minority identities must not be overlooked.

\section{Lucy CJ White,}

MSc Student, Brighton and Sussex Medical School, Brighton, UK.

\section{Max Cooper,}

Senior Lecturer in Primary Care and Public Health, Brighton and Sussex Medical School, Brighton, UK.

\section{David Lawrence,}

HIV and Sexual Health Specialty Trainee, currently working on clinical research in Southern Africa.

\section{Provenance}

Freely submitted; externally peer reviewed.

\section{Competing interests}

The authors have declared no competing interests.

DOI: https://doi.org/10.3399/bjgp19X700349

\section{ADDRESS FOR CORRESPONDENCE}

Lucy CJ White

Brighton and Sussex Medical School, 94 N-S Road, Falmer, Brighton, BN1 9PX, UK.

Email: LCJWhitedoutlook.com

\section{REFERENCES}

1. Alessi EJ, Kahn S, Van Der Horn R. A qualitative exploration of the premigration victimization experiences of sexual and gender minority refugees and asylees in the United States and Canada. J Sex Res 2017; 54(7): 936-948.

2. Alessi EJ. Resilience in sexual and gender minority forced migrants: a qualitative exploration. Traumatology 2016; 22(3): 203-13.

3. Gowin M, Taylor EL, Dunnington J, et al. Needs of a silent minority: Mexican transgender asylum seekers. Health Promot Pract 2017; 18(3): 332-340.

4. Hopkinson RA, Keatley E, Glaeser E, et al. Persecution experiences and mental health of LGBT asylum seekers. J Homosex 2016; 64(12): 1650-1666.

5. Logie $\mathrm{CH}$, Lacombe-Duncan A, Lee-Foon N, et al. "It's for us -newcomers, LGBTQ persons, and HIV-positive persons. You feel free to be": a qualitative study exploring social support group participation among African and Caribbean lesbian, gay, bisexual and transgender newcomers and refugees in Toronto, Canada. BMC Int Health Hum Rights 2016; 16(1): 18.

6. Piwowarczyk L, Fernandez P. Sharma A. Seeking asylum: challenges faced by the LGB community. J Immigr Minor Health 2017; 19(3): 723-732.

7. Fazel M, Wheeler J, Danesh J. Prevalence of serious mental disorder in 7000 refugees resettled in western countries: A systematic review. Lancet 2005; 365(9467): 1309-1314.

8. Lewis NM. Mental health in sexual minorities: recent indicators, trends, and their relationships to place in North America and Europe. Health Place 2009; 15(4): 1029-1045.

9. Alessi EJ, Kahn S, Chatterji S. "The darkest times of my life": recollections of child abuse among forced migrants persecuted because of their sexual orientation and gender identity. Child Abus Negl 2016; 51: 93-105.

10. Lee EOJ, Brotman S. Identity, refugeeness, belonging: experiences of sexual minority refugees in Canada. Can Rev Sociol 2011; 48(3): 241-274.

11. Heller P. Challenges Facing LGBT Asylumseekers: the role of social work in correcting oppressive immigration processes. J Gay Lesbian Soc Serv2009; 21(2-3): 294-308.

12. Doctors of the World. Closing the gaps in healthcare access: the United Kingdom. 2017. https://mdmeuroblog.files.wordpress. com/2018/03/dotw_uk_a5_leaflet_aw1.pdf laccessed 5 Dec 2018).

13. Brooks H, Llewellyn CD, Nadarzynski T, et al. Sexual orientation disclosure in health care: a systematic review. Br J Gen Pract 2018; DOI: https://doi.org/10.3399/bjgp18X694841 